\title{
Research on Big Data Analysis of Location Service and Intelligent Service Platform of Urban Scenic Spots: A Case Study of Guilin City, Guangxi, China
}

\author{
Baishou Li ${ }^{1,2}$, Mingzhi Liao ${ }^{1,}$, Lulu Huang ${ }^{2}$ \\ ${ }^{1}$ College of Geomatics and Geoinformation, Guilin University of Technology, Guilin, Guangxi, China - (lbszhb@163.com, \\ 624463148@qq.com) \\ ${ }^{2}$ Guangxi Key Laboratory of Spatial Information and Geomatics, Guilin, Guangxi, China - lbszhb@163.com,1171575495@qq.com
}

KEY WORDS: big data analysis, location service, tourism scenic spot, platform, intelligent service.

\begin{abstract}
:
In many famous tourist cities, there is a lack of big data analysis and perception of tourist behavior, which reflected in the existence of a large number of basic data in the scenic area. Through the traditional and/or non-special sensors of the Internet of Things, a large amount of special -temporal change data is collected, including video monitors data, RFID, WIFI, temperature and humidity, water depth sensors and other big data of the Internet of Things(IoT)that can perceive the location and environmental resource information of tourists. In addition, the thermodynamic data of the distribution location of tourists' mobile phones, although these data include the behavior data of tourist groups and individuals in the scenic spot, which can be most shown by supplying, lack of in-depth analysis and intelligent service application.

As we all know, Guilin, Guangxi is a famous tourist attraction in the word. In this tourism city, the big data processing methods of location service include establishing a data processing framework, information extraction, fusion and batch processing technology. As a result of the location data of tourists in the scenic spot are constantly created over time, the location service data will be processed by stream mode. At the same time, dimension reduction analysis of big data is an important link in processing as it has a large volume but a low-value density. The analysis methods of location service include kernel density analysis, GIS spatial and temporal analysis, artificial intelligence deep learning, two-dimensional mapping, visualization processing results, and extraction of hot spots or scenic spots, etc. Spatial and temporal index technology is used to manage the big data of scenic spot location service and to improve the efficiency of data query and access. At the same time, a reasonable predictive analysis of data processing results is an important research method.

The main system structure of an intelligent service platform includes platform, data processing sing, and analysis end, mobile management end. The platform includes a general user service module, location service module, a communication module, etc, which is responsible for providing reasonable service to customers. The data processing and analysis end include a data receiving module and a data processing and analysis module. Responsible for receiving and processing the location service data returned by the client. The mobile management end includes the scenic spot management module. Responsible for providing the manager with the distribution of tourists in the scenic area, the location information of the staff, the location of the emergency situation and other contents, and providing great help for the manager to maintain the order of the scenic area, reasonably dispatch personnel, and launch rescue in time. Development and design to show users good models and development concepts and typical artificial intelligence products, to improve the scenic spot tourist's playing efficiency and humanized experience and reduce the management cost.
\end{abstract}

\section{INTRODUCE}

In 2013, the national tourism administration proposed the concept of smart tourism, and many tourism cities in China responded positively and started to take smart tourism as the theme to guide and drive the construction and development of smart scenic spots. But, at present ,in our country, most tourist cities are still Lack of big data analysis and perception of tourist behavior in scenic spots. The acquisition of these information needs to be built on the basis of tourist location acquisition, data processing and analysis. Although these data are currently available through wechat vendors, they lack in-depth analysis and intelligent service applications.

In era of widespread smartphone's use, it is not difficult to obtain real-time location information of tourists. When you use that location information, it's a better place to look. Therefore, this paper constructs the big data analysis and intelligent service platfrom of urban scenic spot location service.Through a variety of big data analysis methods too extract valuable information,so as to provide humanized services for the users of the platform.

\section{WAYS FOR THE PLATFORM TO OBTAIN LOCATION INFORMATION}

\section{1 principle of smart phone positioning}

Mobile phone positioning is a location service based on the positioning module of smart phone. It mainly uses the operators that provide mobile communication services to obtain the corresponding positioning information in the network positioning data and then display it through electronic map, which is a value- added service. The main principle is: when the intelligent machine calls this function, it measures different pilot signals to obtain its corresponding TOA (arrival time), calculates the coordinates according to the final measurement results of the above operations, and finally calculates the

\footnotetext{
* Corresponding author

E-mail addresses: 624463148@qq.com
} 
position to be displayed in the electronic graph by using the trigonometry formula. In the process of mobile phone location, location information acquisition needs to be considered in at least 3 or more than 31 base stations. In the positioning measurement, the positioning accuracy is related to the number of base stations, the more the number of base stations, the higher the positioning accuracy, the better the positioning performance.

\section{2 mobile phone positioning mode}

There are four ways to locate a mobile phone: (1) satellite positioning. also known as GPS positioning. GPS positioning has the advantages of higher positioning accuracy, better positioning performance, rapid positioning, less environmental impact. However, its disadvantages are also obvious. When the weather is not good or it is in a closed environment, the GPS positioning accuracy will be attended to some extent and Based Service for short. (2).Base station location is also called mobile location service.LBS is to obtain the Location information of smart phone users through the operator network providing mobile communication services. At last, it combines with electronic map to provide corresponding services for users using mobile communication services. The advantage of LBS positioning is that it is cheap and low cost.In theory, only the signal difference of three base stations is needed to complete the positioning. That is to affected by the weather, and the indoor environment can still work.However, the disadvantage is that LBS positioning accuracy will change with the number of base stations received by mobile phones (3)Wifi location. Wifi location is to obtain the MAC address of wireless routing devices within a certain range around and the strength of corresponding Wire signal, and then to match through the network in the server high, but if there is no wireless routing equipment, this positioning method cannot be used (4) AGPS positioning. AGPS positioning is a real-time positioning technology combining GPS positioning and base station positioning It can improve the speed of traditional GPS positioning by obtaining the positioning information of mobile phone base station, and use the base station positioning function when GPS satellite signal cannot be observed.

After comparing the above four mobile phone positioning methods, the platform adopts AGPS positioning mode by default. Because the AGPS positioning mode can continue to work in the base station positioning mode when the mobile phone terminal cannot obtain the GPS satellite signal, and once the mobile phone terminal successfully searches the star, the positioning mode will automatically switch to GPS positioning. When the users detected entering the indoor environment, although the AGPS positioning method is also applicable in their door environment, the positioning accuracy is not as high as that of WiFi. In order to improve the positioning accuracy of the mobile phone terminal, the purpose of providing accurate positioning information can be achieved. Therefore, when the platform detects the user entering the room, it will automatically switch the positioning mode to WiFi positioning.

\section{PLATFORM ARCHITECTURE}

main system structure of the big data analysis and intelligent service platform of urban scenic spot location service includes platform end, mobile management end and data processing and analysis end.

\subsection{Data preprocessing}

Platform end is the part provided for tourists in scenic spots, which includes location service module,communication module, general user service module and so on. Among them, the location service module is responsible for selecting the appropriate positioning method according to the specific situation of users, and obtaining the real-time location information with the highest accuracy. The specific method is that when the user is outdoors, the system adopts AGPS positioning. When the user is detected entering the indoor environment, the module first detects the WiFi signal in the indoor environment. If there is, the positioning mode will be switched to WiFi positioning. Precise real-time location collected by location service module.The data will be uploaded to the data processing and analysis end of the intelligent service platform through the communication module And the communication module monitors and receives the processing results return by the data processing end. Finally through the general user service module to provide users with the corresponding humanized services.

\subsection{Mobile management terminal}

The mobile management side is a mobile terminal that is provided to the manager of the area and the rescue personnel, similar to the platform end structure, including the location service module, the communication module, and the specific user service module. The location service module is similar to the communication module and the platform end function, while the difference between the mobile management and the platform is the specific user service module. The module is responsible for the distribution of visitors in the scenic area,the location of the staff, the location of the emergency, and the information data used by the personnel, which is responsible for the maintenance of the order of the scenic area, the reasonable dispatch and the timely launch of rescue has provided great help

\subsection{Data processing analysis}

The data processing analysis of the cloud server of the analysis end is responsible for receiving, storing, managing and processing real-time location service data returned by the client, and real-time data from the depth sensor and temperature and humidity sensors that are distributed evenly across the scenic area. The data processing analysis module includes the communication module and the data processing analysis module where the communication module is responsible for receiving the real-time location service data returned by the client and the inside of the scenic area, and USES the space-time index technique to conduct the management and storage of the location service of the scenic area,and improve the query and access efficiency of the data. The data processing analysis module will extract the real-time location service in the storage space and use the corresponding big data analysis algorithm , and the information mining is carried out. In the scenic area,the real-time data of the location data of the tourist and the sensor in the scenic area are constantly created over time, so the service data for the service data will be handled by the flow processing mode.At the same time, the large number is large but the value density is low, so the reduction of the large number is the important link in the processing. The information of the above processing can be analyzed by the analysis of nuclear density, GIS space time analysis, artificial intelligence depth learning, etc. The results of the final phase are sent to the corresponding client through the communication module. 


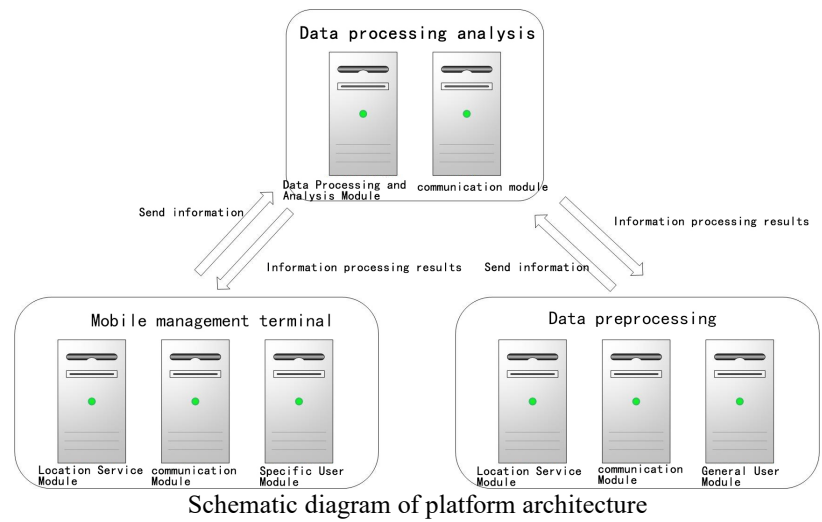

4. SERVICE MODULE

Service module is divided into general user service module and specific user service module. The general user service module is a functional module provided to tourists, while the specific user service module is a functional module serving staff in the scenic spot.

\section{1 general user service}

The general user service module is defined in the intelligent service platform client used by tourists, which is responsible for providing intelligent scenic spot services for tourists. Its functions generally include:

(1) danger warning: when tourists are near the dangerous area, the client will immediately give an alarm and prompt the user, and at the same time inform the user of the distance from the dangerous area to itself and the risk type and other information. The main purpose of this functions to ensure the safety of tourists and prevent them from unknowingly stepping into dangerous areas.

(2) scenic guide route: planning scenic guide route for tourists through real-time positioning information of tourists and rational distribution of terrain of scenic spots and dangerous areas of scenic spots, so as to improve tourists' experience.

The density of tourists in the scenic area is displayed. Through analyzing the real-time location data of all tourists in the scenic area, the heat map of tourists distribution is obtained, so that users can know the real-time density of tourists in each scenic spot in the scenic area, and provide reference for them to plan their own sightseeing route.

\section{2 specific user service}

Specific user service modules are defined in the scenic area in the intelligence service platform client staff use, for the scenic area administrator and rescue personnel to provide intelligent services, its function is roughly include: (1) emergency location: once the visitors too close to the dangerous areas, or in other ways for alarm information, the administrator on the map will be highlighted in the exact location and specific types of emergency, staff name, contact information. According to the above information, the administrator can greatly improve the accuracy, rationality and efficiency of teaching assistance.(2) rescue route planning: once emergencies are confirmed, this function will intelligently plan the safest and most efficient rescue route for rescuers according to real-time tourist distribution heat map and other information within the scenic area. It reduces the time it takes rescuers to find a route and the likelihood that they won't be able to get to the scene in time because of congestion.Improve rescue efficiency. (3)rescue plan: this function provides the rescue plan of various types of emergencies and the warning information and rescue workers to prepare for rescue operations

(4) visitor management: through the access control management by RID technology, the number of tourists in the scenic spot on that day can be obtained.And the number of visitors is too large, the administrator immediately notify stop ticket sales. At the same time, if necessary, the administrator can input the visitor number, id number, mobile phone number and other information, check the case to the designated capacity, and obtain its location and other relevant information for use.

(5) personnel scheduling: through this function, the administrator can get the distribution of all on-the-job staff in the scenic spot.Once an emergency occurs in a certain area, the administrator can reasonably dispatch staff to deal with it according to the above information.

(6) information release: the administrator can use this function to edit the

"announcement" in the intelligent service platform, so that tourists can know all kinds of real-time information about the scenic spot. Such as the performance schedule,temperature, weather forecast and even a missing person notice, find a thing notice.

\section{THE PROCESSING METHOD OF THE LOCATION}

(1)The real-time processing time of real-time location data is typically second or second, usually with real-time flow processing technology. Currently, storm technology is mainly used for flow processing. The technology USES the message flow processing model, which is generated in multiple components and ensures the timeliness of the data processing. The technology is widely used in real-time analysis and online machine learning: continuous computing and distribution. For batch data, real-time requirements are not strict, but you can use spark technology to complete batch tasks when you need to do real-time analysis. The technology can support distributed function processing,Fully play the computing performance of distributed processing and replace map reduce for batch task. At present,select the smallest event: the processing window 0.5 2.s has been able to meet most real-time batch computing scenario requirements. [2] the analysis method of nuclear density is to find the concentration of the target points in the environment by calculating the density of the specified target in its surrounding environment. Based on this feature, this algorithm can be used to analyze the large number of scenic spots in the scenic area, and the best setting of the tourist supply station, the time distribution of the crime in the scenic area, the spatial and temporal distribution of the scenic area, and the easy congestion area Information. Therefore, it provides important reference for the construction and renovation of scenic spots.

The analysis method of nuclear density is to find the concentration of the target points in the environment by calculating the density of the specified target in its surrounding environment. Based on this feature, this algorithm can be used to analyze the large number of scenic spots in the scenic area, and the best setting of the tourist supply station, the time distribution of the crime in the scenic area, the spatial and temporal distribution of the scenic area, and the easy congestion area.Thus provides the important reference for the scenic spot construction and the transformation.

3 ) deep learning of spatial and temporal big data of scenic spots through artificial intelligence deep learning algorithm. So as to predict the occurrence of natural disasters in scenic spots. Supervised learning is an artificial intelligence deep learning algorithm that deduces a hypothesis function from a set of 
manually labeled data. In this learning mode, a large number of training samples constitute a sample set, and each training sample contains input value and expected output value.The deep learning algorithm builds a model based on the above data, and then compares the prediction results generated by the model with the real value, and continuously adjusts the model according to the deviation between the prediction results and the real value, so as to improve the accuracy of the prediction results of the model.

\section{SCENIC SPOT CONSTRUCTION}

In order to maximize the use of intelligent service platform in scenic spots, scenicspots need to be built according to system requirements.

(1) as the ancient saying goes, 'guilin's landscape is the best in the world" Guilin is famous for its landscape,and most of its scenic spots are dominated by landscape Due to the shelter of mountains and trees, many environments may appear Mobile phones can not receive the GPS signal or the signal from the base station in the outdoor, leading to a large increase in outdoor positioning errors. Therefore, the number of base stations should be appropriately increased for scenic spots far away from urban areas and located in remote areas with numerous alpine trees. Even if the phone cannot receive GPS signals, it can still use base station positioning technology to obtain more accurate real-time location information.

(2) the loss of mobile phone signals can be easily caused after entering large indoor environment In response to this situation, the intelligent platform adopts the setting that WiFi positioning can be used when entering indoor environment. Therefore, stable WiFi signal source is very important. Therefore, WiFi base stations should be evenly distributed in large indoor environments in scenic spots, so as to obtain accurate real-time indoor location information of users. In addition, because tourists are usually unfamiliar with the environment, it is easy to get lost or even lost in large indoor environment. Therefore, the scenic spot also needs to provide vector map data of indoor environment. The intelligent service platform combines accurate real-time indoor positioning data with vector map data. Provide indoor location service for users, so as to avoid the occurrence of such incidents.

(3)There are two rainy seasons in guilin. One is the monsoon season, which falls in March and may,accounting for about half of the year's $1,900 \mathrm{~mm}$ of rainfall. The second is from June to August, when guilin is prone to flooding, And guilin scenic area and most have rivers and lakes and so on.environment and risk easily happened during the rainy season, so with the environment of the scenic area, such as rivers and lakes shall be placed in such an environment water depth sensor, and set the depth of threshold, once detected water depth more than threshold, immediately send information to the intelligence service platform, only to remind visitors to carefully. At the same time,temperature sensors are evenly distributed in the scenic area. Once the surface temperature is detected to be too high for tourists, corresponding warnings will be issued.

(4) replace traditional paper tickets with electronic tickets with RFID technology, and install RFID identification equipment.The tickets can be printed out or printed into CARDS, and existing technology only Can achieve access control management. On the one hand, fake tickets can be detected; On the one hand, fake tickets can be detected; on the other hand, the labor cost of the scenic spot can be reduced and the efficiency of scenic spot staff can be improved. For large scenic spots, it usually takes two to three days for tourists to visit each scenic spot and enter each scenic spot for several times. The traditional paper ticket is easy to cause the phenomenon of multi-use of one ticket or reuse of fake or invalid tickets. The electronic ticket produced by the scenic spot with RFID technology can easily eliminate the above problems.

\section{CONCLUSION}

The big data analysis and intelligence service platform of the location service of urban tourism scenic spots improves the accuracy of real-time location data obtained by automatically switching location method according to the user's environment. The aim of providing precise services has been achieved.

This design USES big data processing and analysis methods to conduct information mining for a large number of dynamic realtime location service data, so as to achieve in-depth analysis and 1 intelligent service application.

According to different user's identity and function at the same time, according to the real-time location data after big data processing results, tailored personalized service projects, in order to meet the visit scenic spot of tourists and improve efficiency and human experiences, and to safeguard the order of the scenic area, reasonable scheduling, timely launch aid provided convenience.

\section{ACKNOWLEDGMENTS}

This work was supported by the Guangxi Nature Science Foundation (No.2016GXNSFAA380013);Guilin Scientific Research and Technology Development Plan(20190601 ; 2016012601),the Innovation and Entrepreneurship Training Program of Chinese College Students (NO.201910596632)

,Guilin Guilin University of Technology Ph.D Scientific research initial funding(002401003316);Chongqing basic science and advanced technology research (cstc2015jcyjBX0023).Special thanks to the College of Geomatics and Geoinformation, Guilin University of Technology for the support of our work.

\section{REFERENCES}

Zhou jin., 2019.deep learning algorithm of artificial intelligence.Computer program skills and maintain. (07):115117.

Wu binkun., 2019.Big data analysis and application of mobile Internet[J].Information and computer(theoretical edition) (12):180-182.

Jie qiao., 2019.the research of urban museum groups basedon uncleardensity analysis-take the planing research of museum groups in nanhai district,foshan city as an example [J].intelligent city ,5(11):27-28.

Xie guang,Zhong cheng.,2018. research on mobile phone positioning method and accuracy $[\mathrm{J}]$. Modern information, 2(07):36-37+40.

Liu kang, sun yun, ning ru.,2016.Rfid- based intelligent scenic spot construction $[\mathrm{J}]$. Prospect of science and technology, 26(35):1-2. 